\title{
Analysis of bacteria-challenged wild silkmoth, Antheraea mylitta (lepidoptera) transcriptome reveals potential immune genes
} Archana S Gandhe ${ }^{\dagger}$, K P Arunkumar ${ }^{\dagger}$, Serene H John and J Nagaraju*

Address: Laboratory of Molecular Genetics, Centre for DNA Fingerprinting and Diagnostics, ECIL Road, Nacharam, Hyderabad 500076, India

Email: Archana S Gandhe - archana@cdfd.org; K P Arunkumar - arun@cdfd.org.in; Serene H John - hserene@cdfd.org.in;

J Nagaraju* - jnagaraju@cdfd.org.in

* Corresponding author †Equal contributors

Published: 2I July 2006

BMC Genomics 2006, 7:184 doi:10.1|86/|47|-2|64-7-184
Received: 04 April 2006

Accepted: 21 July 2006

This article is available from: http://www.biomedcentral.com//47/-2/64/7//84

(C) 2006 Gandhe et al; licensee BioMed Central Ltd.

This is an Open Access article distributed under the terms of the Creative Commons Attribution License (http://creativecommons.org/licenses/by/2.0), which permits unrestricted use, distribution, and reproduction in any medium, provided the original work is properly cited.

\begin{abstract}
Background: In the recent years a strong resemblance has been observed between the insect immune system and the mammalian innate immune mechanisms suggesting their common origin. Among the insects, only the dipterans (Drosophila and various mosquito species) have been widely investigated for their immune responses towards diverse pathogens. In the present study we constructed and analysed the immune transcriptome of the lepidopteran Antheraea mylitta, an economically important Indian tasar silkmoth with a view to unravel the potential immune-related genes and pathways.

Results: An expressed sequence tag (EST) library was constructed from mRNA obtained from fat bodies of $A$. mylitta larvae that had been challenged by infection with Escherichia coli cells. We identified 719 unique ESTs from a total of 1412 sequences so generated. A third of the transcriptome showed similarity with previously characterized immune-related genes that included both the known and putative immune genes. Of the four putative novel defence proteins (DFPs) annotated by PSI-BLAST three showed similarity to extracellular matrix proteins from vertebrates implicated in innate immunity, while the fourth was similar to, yet distinct from, the anti-microbial protein cecropin. Finally, we analysed the expression profiles of 15 potential immune-related genes, and the majority of them were induced more prominently with E. coli compared to Micrococcus luteus. We also identified several unknown proteins, some of which could have probable immunerelated functions based on the results of the ProDom analysis.
\end{abstract}

Conclusion: The present study has identified many potential immune-related genes in A. mylitta some of which are vertebrate homologues and others are hitherto unreported putative defence proteins. Several genes were present as members of gene families, as has also been observed in other insect species.

\section{Background}

Insects are evolutionarily successful organisms and occupy almost all habitats in nature. An efficient immune system is one of the attributes for this evolutionary success. However, unlike mammals, the insects lack an adap- tive immune system. The insect immune response is comprised of cellular and humoral components. The former involves the action of haemocytes in phagocytosis of microbes, encapsulation of large pathogens and nodule formation [1] whereas the latter involves activation of 
prophenoloxidase cascade leading to melanisation of invading microorganisms [2] and synthesis of a battery of anti-microbial peptides [3].

Insect immunity is well studied in dipterans such as fruit flies and mosquito species [4-7]. Only limited information is available on genes induced on pathogen challenge in a few lepidopteran species that include the domesticated silkmoth, Bombyx mori [8], Cecropia moth,Hyalophora cecropia [9] and tobacco hornworm,Manduca sexta [10] and in these too the immune response pathways employed to combat pathogen infections remain to be fully characterised.

Abundant genetic resources are now available for B. mori, with a $9 \mathrm{X}$ shotgun sequence coverage of its genome and more than 100,000 ESTs in dbEST (NCBI) [11-13]. With reference to insect immunity, the ESTs have been obtained from baculovirus-infected $B$. mori cultured cells and pupae, but no large scale information on bacteria-induced immune genes is as yet available.

In this study, we have constructed and analysed an immune transcriptome following bacterial challenge of the Indian tasar wild silkmoth, Antheraea mylitta, an economically important lepidopteran cultivated for silk production. Prior information on immune response genes in wild silkmoths is lacking except for a few peripheral studies. Two proteins from A. mylitta - a lysozyme protein, 3$\mathrm{D}$ structure of which is elucidated [14] and a protease inhibitor have been characterized [15]. We chose to examine the fat body transcriptome since it is a major immune organ in insects, analogous to the mammalian liver. We generated a total of 1412 ESTs, of which $31 \%$ could be ascribed to putative immune functions. We also validated the upregulation of a selected subset of genes from the immune transcriptome by semi-quantitative RT-PCR.

\section{Results and discussion}

As described in Materials and Methods, we constructed a cDNA library from fat body tissues of $E$. coli- challenged $A$. mylitta larvae and randomly sequenced a large number of inserts from the library. By running TGICL program, we obtained 719 clusters from a total of 1412 ESTs, of which 166 were contigs (comprising 859 ESTs) and 553 were singletons. The majority of the EST clusters were 500 to $600 \mathrm{bp}$, with an average of $524 \mathrm{bp}$ and a maximum of 1994 bp (Figure 1). Each of these clusters potentially represents a unique gene from $A$. mylitta, and our results have hence expanded the number of genes known for this organism from the handful previously known genes. The 1412 EST sequences (accession numbers EB742119EB743530) can be accessed at the NCBI EST sequence database, dbEST and the 719 clusters can be accessed at URL mentioned in references [16].

\section{BLAST analysis}

The different ESTs were classified into categories such as immune-related, housekeeping, hypothetical insect proteins, hypothetical non-insect proteins based on the homology in NCBI protein BLAST (Table 1). Of the 1412 ESTs, $432(31 \%)$ showed similarity to known or putative insect immune proteins; thus, even though the cDNA library was not normalized, a significant proportion (onethird) of the transcriptome was represented by putative immune-related genes. A total of 569 ESTs (39\%) were homologous to housekeeping genes and proteins involved in functions other than immunity. Functional class distribution of the ESTs is depicted in Table 1. Based on the sequence similarity, 679 of the 1412 ESTs were classified as insect-specific (224 of the 719 clusters) and 409 ESTs (204 clusters) were common to both insects and mammals (Figure 2a and 2b). The number of ESTs showing similarity to only the mammalian proteins was 14 and those showing similarity neither to insects nor to mammals were as few as 13. Gene Ontology analysis of the EST sequences was also carried out and the details are provided as supplementary data (see Additional data file 1).

\section{Domain search}

The sequences, which could not be assigned any function based on homology search in NCBI, were searched for conserved domains in ProDom database. Of the 260 clusters that had no matches with known proteins in NCBI BLAST, we could assign protein domain families to 196 clusters based on the ProDom search (see Additional data file 2). The remaining sequences did not show any hits in ProDom and should be further analysed by other specialized computational tools.

\section{Signal peptide and transmembrane domains}

We further screened the unannotated proteins (no hits or hits with hypothetical proteins in NCBI database) for the presence of signal peptide and absence of transmembrane domains. This characteristic of many of the immune proteins has been utilized to screen probable immune-related genes in large-scale transcriptome studies [17], although the reliability of this criterion to identify the immune related genes remains to be experimentally tested. In the present study, 25 out of 260 genes tested fulfilled these criteria and could be considered as potential immuneresponsive genes. However, since all the 260 clusters checked are not all full-length sequences, it is possible that we may have missed some others amongst them that represent gene products harbouring signal peptide without transmembrane domain, and hence the actual number may be higher.

\section{Putative immune proteins}

A total of 80 clusters were assigned a putative immune function based on their similarity with previously charac- 


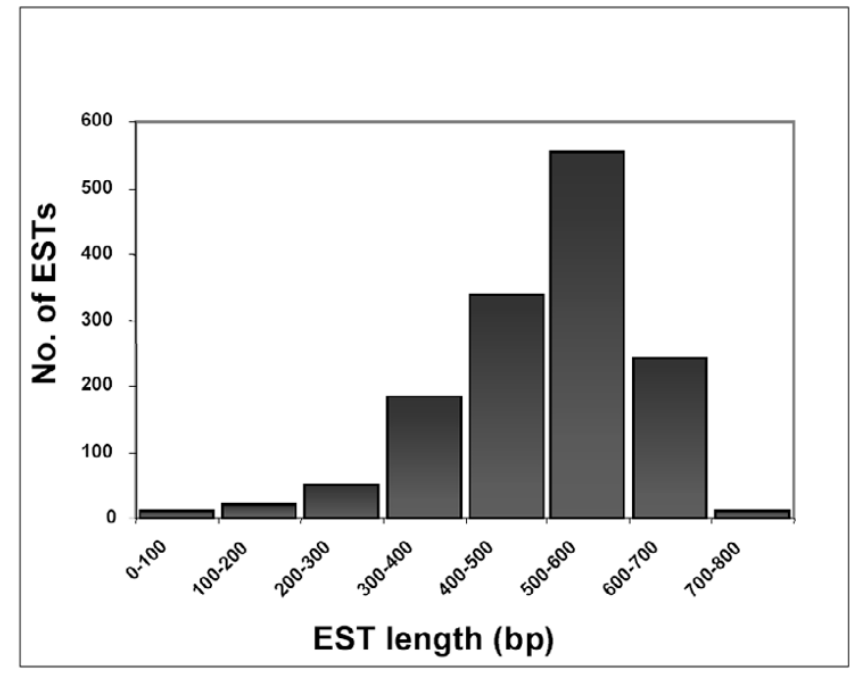

(a)

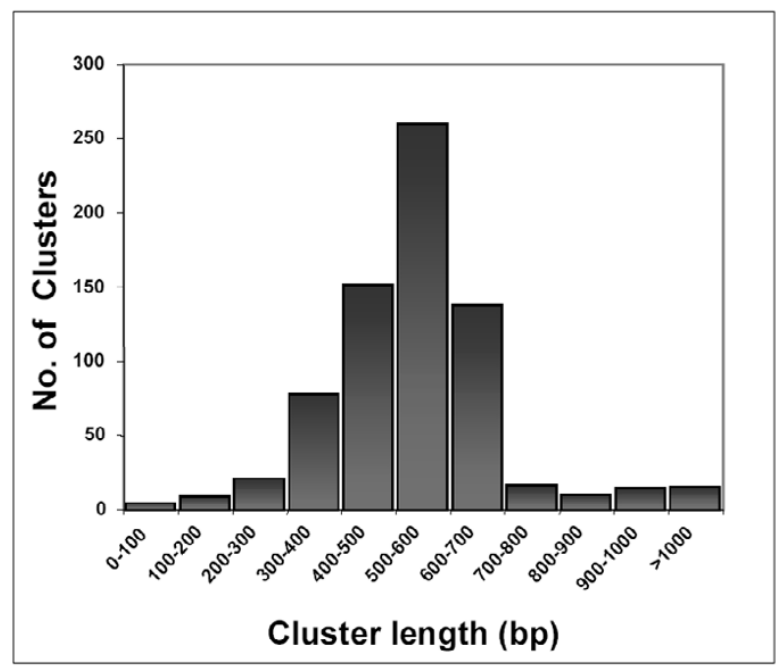

(b)

Figure I

Distribution of read lengths of $A$. mylitta ESTs and clusters. (a) Read lengths of I4I2 ESTs. (b) Read lengths of clusters.

terized immune response genes and their distribution pattern is shown in Figure 3. These putative immune proteins were categorized into different functional groups - i. antimicrobial proteins, ii. pattern recognition receptors (PRRs), iii. proteases and protease inhibitors and iv. putative defence proteins with unknown function. A subset of these putative immune genes (thirty-eight) is represented with the details like GenBank accession numbers, putative function, E-value (BLAST) etc (see Additional data file 3). Homologues of several other proteins, implicated in immunity such as antioxidants, ferritins, transferrins, apolipophorins and apoptotic pathway components were also found, but are not further described here.

\section{Putative anti-microbial proteins}

Attacin-like proteins were the most abundantly expressed transcripts in terms of EST copy number in the immune transcriptome (Table 2). Six different types of putative attacins accounted for 34\% (148 ESTs) of the immune transcripts. A previous study had reported the presence of four types of attacins in D. melanogaster [18] and a similar study in immunized pupae of $H$. cecropia led to the isolation of six closely related types of attacins [9]. Second in the list of highly expressed genes were cecropin-like proteins (55 ESTs, 13\%). Three types of cecropin homologues were found in the A. mylitta transcriptome and a previous study in $H$. cecropia had similarly shown the existence of three types of cecropins [19]. Three types of putative lysozyme transcripts were detected, one of which was homologous to a bacteriophage T7 lysozyme-like protein reported in $B$. mori that was later named, based on functional analysis, as a peptidoglycan recognition protein [20]. The second was homologous to the bacteriolytic lepidopteran lysozymes known to lyse the bacterial cell wall [21]. The third was a lysozyme-like protein that lacks one or both of catalytic residues essential for muramidase

Table I: Classification of EST functional class categories based on similarity searches with NCBI protein database.

\begin{tabular}{lc}
\hline Category & No. of ESTs \\
\hline Total no. of ESTs & $1412(100 \%)$ \\
Immune-related & $432(31 \%)$ \\
Housekeeping genes and other genes not involved in immunity & $569(39 \%)$ \\
Hypothetical/unknown insect proteins & $135(9.5 \%)$ \\
Hypothetical/unknown non-insect proteins & $50(3.5 \%)$ \\
Weak homology in NCBI* & $170(12 \%)$ \\
No homology in NCBI & $56(3.9 \%)$ \\
\hline
\end{tabular}

Percentage value of each category is in parenthesis. *- Hits with relatively less similarity $(E$ value $>l e-05)$. 

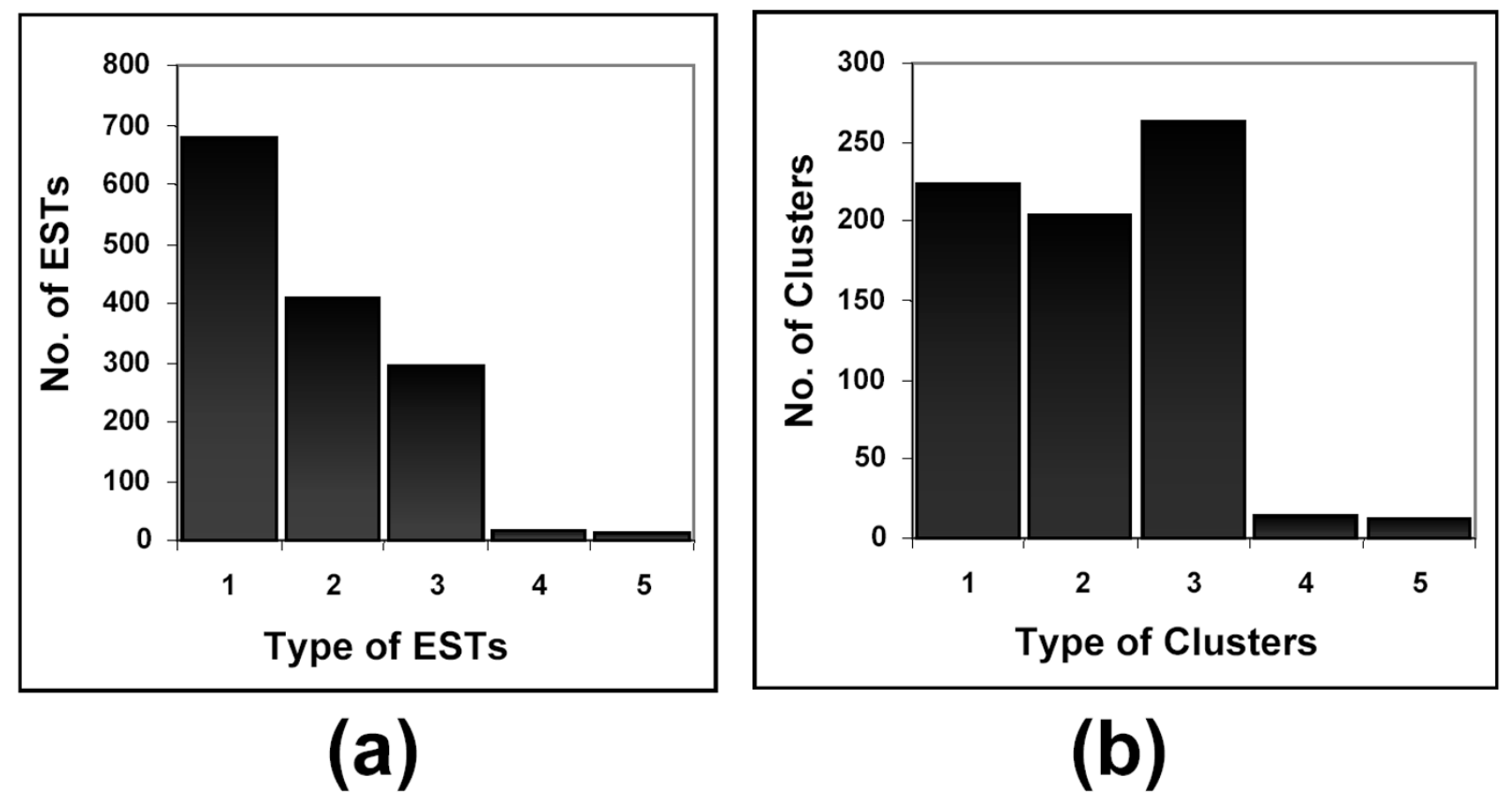

\section{Figure 2}

Classification of ESTs (a) and Clusters (b) based on the number of sequences showing similarity to insect and or mammalian proteins in NCBI protein nr database. X-axis: I) Insect, 2) Insect and Mammal, 3) Unknown, 4) Mammal, 5) Neither Insect nor Mammal, Y-axis: Number of sequences.

activity. This protein has not been reported in lepidopterans before and is homologous to dipteran lysozyme-like proteins of unknown function. Three types of putative lebocins and gloverins, which are the lepidopteran spe-

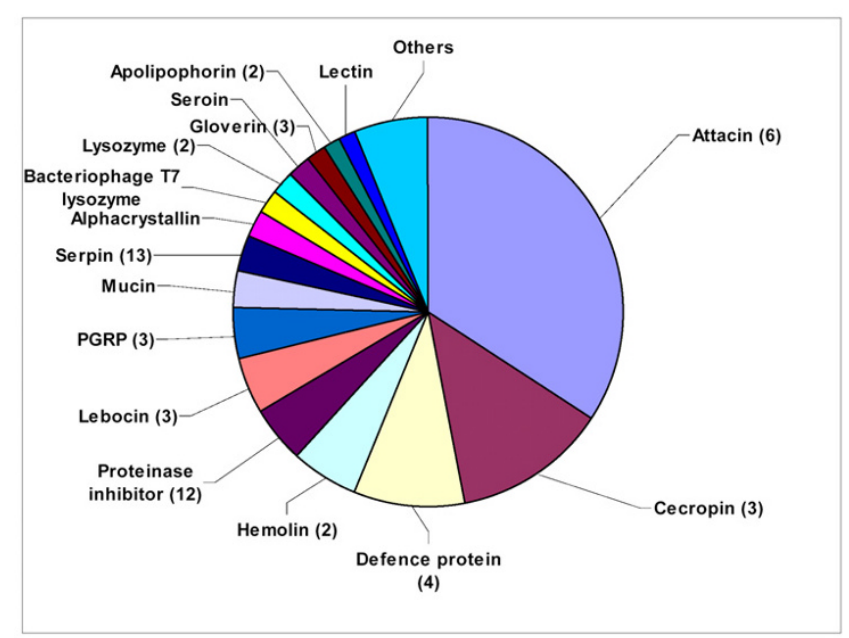

Figure 3

Distribution of immune-related transcripts in A. mylitta fat body transcriptome. The figure in parenthesis indicates the number of isoforms identified in that particular gene family. cific anti-bacterial genes, were also observed in A. mylitta transcriptome.

The data generated in the present study conformed to the trend of anti-microbial genes being present as multiple gene families as observed in many other insect species and highlighted the essentiality of these genes in the organism [22]. Another interesting finding with respect to immune response was the presence of a protein identical to seroin. This protein is reported to express in the silk glands of $B$.

Table 2: The abundant genes in A. mylitta transcriptome.

\begin{tabular}{lc}
\hline Gene definition & No of ESTs \\
\hline Attacin & 96 \\
Cecropin D & 31 \\
Defense protein I & 30 \\
Ribosomal protein S2, Attacin & 26 \\
Elongation factor I $\alpha$ & 24 \\
Hemolin & 23 \\
Attacin & 22 \\
Cecropin & 17 \\
Ferritin & 15 \\
Mucin & 13
\end{tabular}

The number of ESTs indicates the transcript abundance for each of the category 
mori and is known to protect the cocoon from microbes [23]. A homologue of anti-fungal protein, gallerimycin, previously characterized from Galleria mellonella and shown to be induced by LPS injection [24], was also found.

\section{Putative pattern recognition receptors (PRRs)}

PRRs in the insects bind to and detect pathogen associated molecular patterns (PAMPs) like lipopolysaccharide, peptidoglycan, $\beta$ 1-3 glucan, lipotechoic acid etc $[9,25]$. The putative PRRs identified in the present immune transcriptome analysis were hemolins, peptidoglycan recognition proteins (PGRPs), gram-negative binding proteins (GNBPs), lectins and mucins. A. mylitta fat body transcriptome revealed two different proteins resembling hemolin, an immune inducible protein implicated in insect immunity [26] and it would be worthwhile to study the function and specificities of the different proteins of hemolin family. Three types of PGRP-like proteins were found in the $A$. mylitta immune repertoire in the present study as compared to $D$. melanogaster where 13 PGRP genes are known to be involved in the activation of the various effector pathways in immunity [27].

\section{Putative proteases and protease inhibitors}

Various proteases and protease inhibitors regulate the diverse immune mechanisms like melanization, phagocytosis and induction of anti-microbial peptides [10]. A homologue of a prophenoloxidase activating protease characterized in B. mori and M. sexta [11] was also found in the A. mylitta immune transcriptome. Several other classes of putative proteases like cysteine proteases, serine proteases and metallo-proteases were also identified. As many as thirteen distinct serpin-like (serine protease inhibitors) and twelve potential protease inhibitors were detected in the immune transcriptome. Five different serpins have earlier been identified in M. sexta and shown to differ in the induction pattern upon immune challenge [1]. In the light of these studies, the information on serpins and protease inhibitors from the A. mylitta immune transcriptome will prove to be invaluable in further understanding of various immune pathways in insects.

\section{Putative defence proteins with unknown function}

Several potential new members of known protein families were identified in the present study. Among the new members, one was a putative lysozyme-like protein described in the previous section. An array of putative proteinase inhibitors and proteases were also found. Many of them are new, and their study would enhance our understanding of the mechanisms of proteolytic cascades in insect innate immunity. A few immunoglobulin (Ig) like molecules were identified by ProDom search (see Additional data file 2). Ig-like molecules- hemolin [26] and more recently Dscam have been implicated in insect immunity $[28,29]$ and it would be interesting to evaluate the role of these putative Ig-like molecules in insects. Among the potential immune proteins, we describe below in more detail four putative defence proteins (DFPs), for two of which (DFP-1 and DFP-4) we have confirmed the induction upon E. coli infection by semi-quantitative RTPCR.

\section{DFP-I, DFP-2 and DFP-3}

We have grouped these three proteins together, as they are $70-85 \%$ similar to each other (DFP-1\&DFP-3 $=85 \%$, DFP- $1 \&$ DFP- $2=79 \%$ and DFP-2\&DFP-3 $=77 \%$ ). All of them have a signal peptide and appear to be secretory proteins. DFP-1 was abundantly expressed in the immune transcriptome suggesting its possible involvement in immunity. In addition, these three proteins showed high similarity to immune induced unknown proteins from other lepidopterans like Hyphantria cunea, Samia cynthia ricini,M. sexta and Lonomia obliqua [10,30-32] (Figure 4), as also with some hypothetical proteins from other insects and vertebrates in the NCBI database (see Additional data file 4). Analysis by Position-specific iterative BLAST (PSIBLAST) [33] revealed similarity of DFP-1, 2 and 3 to the vertebrate extracellular matrix proteins (ECM), stromal cell derived factor receptor-2, spondin and reelin and possessed the common domain termed as 'reeler' (Table 3). These ECM proteins are involved in the central nervous system signaling and immune mechanisms like 'signaling' and 'pathogen recognition' [34,35]. Stromal cell derived factor/receptor complex has been shown to activate JAK/ STAT pathway and mediate the migration and proliferation of haematopoietic cells [35]. Recently, mindin a protein belonging to the F-spondin family has been shown to act as a pathogen recognition receptor in mice [34]. Also, spondin has been shown to be upregulated in Drosophila upon bacterial infection by microarray analysis [36]. The similarity of DFP-1, 2 and 3 to molecules involved in immune responses in vertebrates further support the immune-related role of these new proteins.

\section{DFP-4}

This protein was particularly intriguing as it showed similarity to cecropin, the insect anti-bacterial peptides in the primary sequence BLAST analysis. The multiple alignment of the various cecropins and DFP-4 is shown in Figure 5. Based on the SignalP prediction, DFP-4 is likely to be a non-secretory intracellular protein, unlike cecropins that are secreted into the haemolymph. Cecropins are small 5$6 \mathrm{KD}$ peptides whereas DFP-4 is a $17 \mathrm{KD}$ protein with additional unrelated regions at the $\mathrm{N}$ and $\mathrm{C}$-termini. The exact role of DFP-4 is not clear and needs to be investigated. 

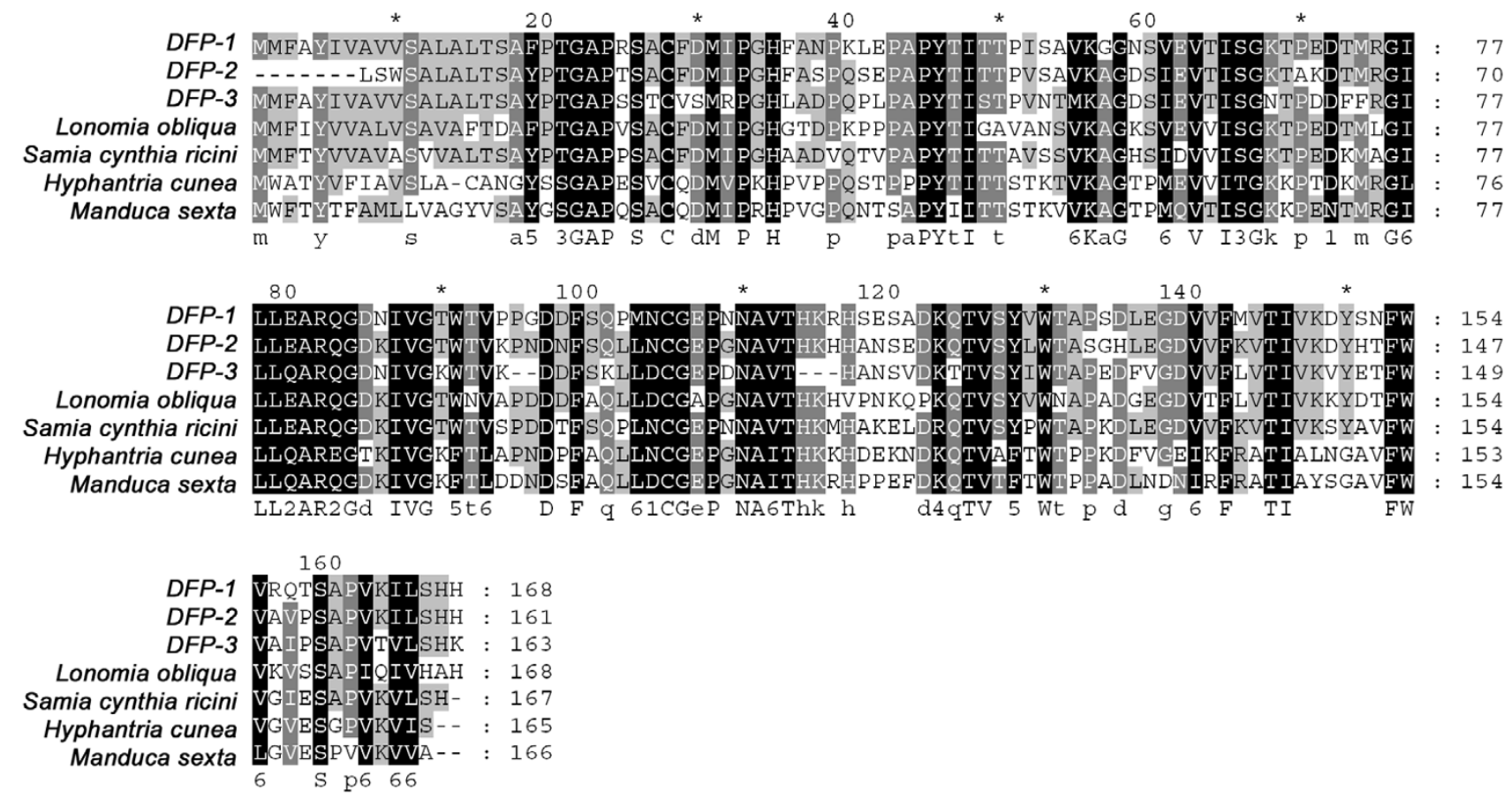

\section{Figure 4}

Multiple sequence alignment of DFP-I, 2 and 3 amino acid sequences with homologues from other lepidopterans. Lonomia obliqua [GenBank:AAV91350], Manduca sexta [GenBank:AAO2I 507], Samia cynthia ricini [GenBank:BAD05929], Hyphantria cunea [GenBank:AAD09280] protein sequences, respectively. Black and grey shades indicate the identical and similar amino acid residues, respectively.

\section{Expression profile}

The transcriptome under study most likely represents a plethora of $E$. coli-induced genes in the fat body of the tasar silkmoth. We validated 15 putative immune response genes by semi-quantitative RT-PCR, and their expression profiles are shown in Figure 6. All but two genes, a putative protease inhibitor [GenBank:DQ666519] and a seroin gene [GenBank:DQ666525] were upregulated upon infection. The two DFPs tested, DFP-1 [GenBank:DQ666501] and DFP4 [GenBank:DQ666503], were induced by both E. coli and M. luteus. DFP-1 was highly expressed in all the tissues

Table 3: Proteins similar to putative defence proteins (DFPs) revealed by PSI-BLAST analysis.

\section{Novel proteins from A. mylitta Description and function of proteins similar to DFPs.}

DFP-I, DFP-2, DFP-3

\section{Stromal cell derived factor receptor 2 homologue \\ Homo sapiens [GenBank:NM 001013660.1] \\ (catecholamine catabolism) reeler domain}

\section{Spondin}

Bos taurus [GenBank:Q9GLX9]

H. sapiens [GenBank:Q9HCB6]

Extracellular matrix, cell adhesion protein that promotes the attachment of spinal cord and sensory neuron cells

\section{Reelin precursor}

H. sapiens [GenBank:P78509]

Extracellular matrix serine protease. Enzymatic activity is important for the modulation of cell adhesion

DFP-4

\section{Cecropin D precursor}

L. obliqua [GenBank:AAV9|462]

The GenBank accession numbers are given in parentheses. 


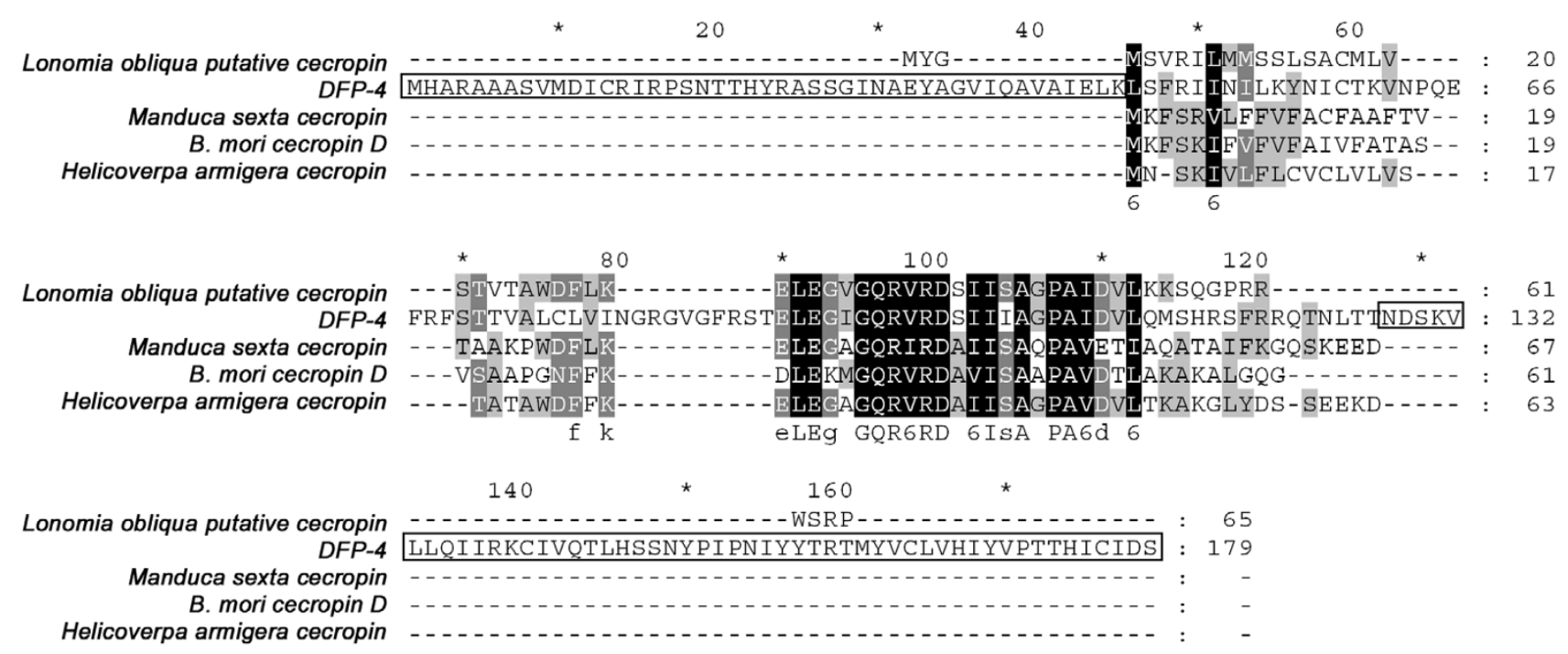

\section{Figure 5}

Multiple sequence alignment of DFP-4 with cecropins from other lepidopterans. L. obliqua putative cecropin [GenBank:AAV9|462], M. sexta cecropin [GenBank:AAO74638], B. mori cecropin D [GenBank:BAE5337I], Helicoverpa armigera cecropin [GenBank:AAX51|93.1]. The unique regions of DFP-4 (no homology to cecropins) are boxed.

with a more prominent expression in mid gut whereas DFP-4 was exclusively expressed in fat body (Figure 6). We analysed the expression pattern of the aforementioned genes in larval tissues differentially challenged with $E$. coli (gram-negative) or M. luteus (gram-positive) and compared with challenged or mock-challenged (salineinjected) tissues as negative controls. Most of the genes analysed were expressed more prominently upon infection with E. coli than with M. luteus suggesting that there may be differential responses towards different pathogens. Two pathways of differential immune induction have been identified in Drosophila. Gram-positive bacteria and fungi induce the Toll pathway whereas gram-negative bacteria evoke the Imd pathway [37], but the degree of conservation of these pathways between Drosophila and lepidopterans is not known.

\section{Conclusion}

The immune response in insects is dynamic and different effector genes are likely expressed at different time points during infection, contributing to the ability of the insects to ward off infections in spite of the absence of adaptive immunity. The current transcriptome represents genes likely expressed upon E. coli infection in the A. mylitta fat body at 24 hrs post infection. Unexpectedly, the Imd pathway components that are implicated in the activation of various effector pathways upon gram-negative bacterial infection in Drosophila were not present in our transcriptome. The 24-hour post infection period may have been non-optimal for expression of some genes, and since the
EST library was not normalized the less abundant transcripts may have gone undetected. Alternatively, it is possible that other pathways are involved in the immune induction in the moths.

The present study has increased the repertoire of lepidopteran-specific putative immune response genes by several hundred-fold. This will be a valuable resource for lepidopteran-specific immune studies in particular and insect immune studies in general.

\section{Methods}

Insects, bacterial inoculation and tissue collection

A. mylitta, $5^{\text {th }}$ instar, day 3 larvae were procured from Regional Research Station, Warangal, Andhra Pradesh. Log phase E. coli cells (DH5 $\alpha$ ), washed and resuspended in saline $(0.3 \mathrm{M} \mathrm{NaCl}, 0.005 \mathrm{M} \mathrm{KCl})$, were injected into the haemocoel of the larvae as described earlier [38]. At 24 hours post infection (hpi), larvae were dissected to isolate fat body, and the tissue was flash frozen in liquid $\mathrm{N}_{2}$ and then stored at $-70^{\circ} \mathrm{C}$ till further use.

\section{cDNA library construction and generation of ESTs}

Total RNA was extracted from the fat body using Trizol reagent (Invitrogen). The complementary DNA synthesis was carried out using Stratagene ZAP-CDNA ${ }^{\circledast}$ synthesis kit following manufacturer's instructions. Directional cDNA library was constructed by cloning of cDNA fragments into pBluescript II SK (+) vector and electroporation into E. coli strain DH10B. Insert-containing plasmid clones 


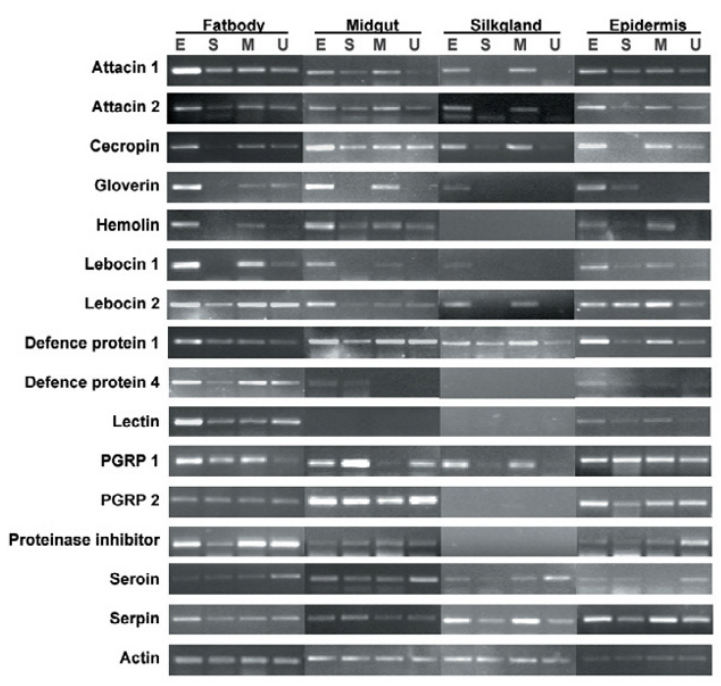

\section{Figure 6}

RT-PCR profiles of 15 putative immune genes from 4 differentially challenged tissues of $5^{\text {th }}$ instar larvae. E- E. coli challenged, M- M. luteus challenged, S- saline-injected, $U$ unchallenged. Actin was used as an endogenous control. The GenBank accession numbers of the 15 genes are provided as additional data (see Additional data file 5).

were sequenced with RV-M primer (5'GAG CGG ATA ACA ATT TCA CAC AGG 3') with the aid of MegaBACE3000 sequencer.

\section{EST processing}

Raw sequences obtained from sequence chromatograms were processed using several programs. A cut-off Phred Quality Value of $\geq 15$ was assigned to extract quality sequences from chromatograms. The quality sequences were screened for the presence of vector sequences using 'Cross Match' program [39]. Then masked vector sequences were automatically removed by in-house developed trimming tool. Sequences shorter than 50 bases were removed. The resulting high-quality sequences were assembled into sequence contigs with the TGICL program [40], which initially makes clusters using MegaBLAST and thereafter makes an assembly using CAP3 for each cluster generated in the first step. A cluster is defined as a unique sequence obtained either by multiple alignment of many sequences that are $>95 \%$ similar or derived from a single sequence. A cluster containing $\geq 2$ ESTs is termed a contig and that containing only one sequence, a singleton.

The unique putative gene sequences obtained by clustering and assembly were annotated by running BLAST [41] against non-redundant $(n r)$ protein database of NCBI. Further, BLAST output was parsed to classify the putative gene transcripts into different functional classes.

\section{Analysis of unknown proteins}

Proteins that did not show any significant hits in NCBI $n r$ database or showed similarity to unknown or hypothetical proteins were characterized by additional computational tools.

\section{a. Domain search}

For the sequences showing high similarity to hypothetical and/or unknown proteins, and those showing weak similarity to $n r$ protein database, domain search was performed using ProDom [42]. Putative function was assigned based on the type of domains found.

\section{b. Signal peptide and transmembrane domain analysis}

Presence of transmembrane domains and signal peptide analysis was done on the transcripts not showing any significant hits in NCBI database. The signal peptide analysis was done by SignalP software [43] and trans-membrane domain analysis was done with TMHMM program [44].

\section{c. Functional annotation with PSI-BLAST}

The functional annotation of four novel immune upregulated transcripts (DFPs) was done using PSI-BLAST [33].

\section{Expression profile}

A. mylitta larvae were differentially challenged- a) Unchallenged, b) Saline-injected, c) M. luteus and d) E. coli. Log phase E. coli and M. luteus bacteria, washed and resuspended in insect saline $(0.3 \mathrm{M} \mathrm{NaCl}, 0.005 \mathrm{M} \mathrm{KCl})$ were injected $(30 \mu \mathrm{l})$ into a set of A. mylitta larvae. One set each of saline-injected and uninjected larvae were kept as a control. Four tissues, namely fat body, epidermis, mid gut, and silk gland were dissected out and flash frozen in liquid nitrogen. Total RNA was isolated using Trizol reagent. To remove genomic DNA contamination, total RNA was treated with RNAse free DNAse (NEB) as prescribed by the manufacturers. cDNA was synthesized using MMLV reverse transcriptase (Invitrogen) and oligo dT primers from $1 \mu \mathrm{g}$ of total RNA. The primers were designed for the selected ESTs by Primer3 software [45].

Semi-quantitative RT-PCR was carried out for all the four differentially challenged tissues using an Eppendorf master cycler under the following conditions- $94^{\circ} \mathrm{C}, 2 \mathrm{~min}$ initial denaturation, 27 cycles $\left(94^{\circ} \mathrm{C}-30 \mathrm{~s}, 58^{\circ} \mathrm{C}-30 \mathrm{~s}\right.$, $\left.72^{\circ} \mathrm{C}-2 \mathrm{mins}\right)$ and a final elongation at $72^{\circ} \mathrm{C}$ for $10 \mathrm{mins}$. Actin cDNA was amplified as an endogenous control. PCR reaction components included: $1 \mathrm{X}$ buffer, $100 \mu \mathrm{M}$ dNTPs, $1.5 \mathrm{mM} \mathrm{MgCl}$ 2, 0.5 units Taq polymerase (MBI), $0.5 \mu \mathrm{M}$ primers. Primer sequences are enlisted in additional data (see Additional data file 5). 


\section{Obtaining full-length cDNA by 5 ' RACE}

We had obtained full-length coding sequences of the DFP1,2 and 3 through EST sequencing. To acquire full-length DFP-4 CDNA, we carried out 5' RACE PCR using the 5' RACE kit (Clontech). The 5' ends were amplified by using an adaptor primer and a reverse gene specific primer. PCR was performed for 25 cycles in an Eppendorf master cycler. A $300 \mathrm{bp}$ band was isolated, sequenced and confirmed to be the 5' DFP-4 sequences.

\section{Authors' contributions}

AG and AKP conceived and designed the study, participated in the bioinformatics and validation analysis, and drafted the manuscript. SH carried out the expression profile analysis, assisted in the bioinformatics analysis and manuscript preparation. JN conceptualised the project, critically reviewed the manuscript and provided guidance. All the authors read and approved the final manuscript.

\section{Additional material}

\section{Additional data file 1}

Additional data file 1 includes the results, methodology and schematic representation of Gene Ontology analysis of the ESTs.

Click here for file

[http://www.biomedcentral.com/content/supplementary/14712164-7-184-S1.pdf]

\section{Additional data file 2}

Additional data file 2 is a table listing the ProDom based annotation of the clusters that did not find any matches in NCBI protein BLAST analysis.

Click here for file

[http://www.biomedcentral.com/content/supplementary/14712164-7-184-S2.pdf]

\section{Additional data file 3}

A table representing some of the putative immune genes from the transcriptome is provided as an Additional data file 3

Click here for file

[http://www.biomedcentral.com/content/supplementary/14712164-7-184-S3.pdf]

\section{Additional data file 4}

Additional data file 4 is a table that lists the hits obtained by NCBI protein BLAST analysis of DFP-1, 2, 3 and 4.

Click here for file

[http://www.biomedcentral.com/content/supplementary/1471-

2164-7-184-S4.pdf]

\section{Additional file 5}

Primer sequences of the 15 transcripts analysed by RT-PCR have been provided in Additional data file 5.

Click here for file

[http://www.biomedcentral.com/content/supplementary/14712164-7-184-S5.pdf]

\section{Acknowledgements}

We acknowledge Ms Riti Mohapatra for her assistance in the bioinformatics analysis. We are thankful to Mr. Riccardo M. Bennett-Lovsey and Mr. Alex D. Herbert, graduate students, Imperial College, London, for their suggestions in annotating the unknown proteins. We also acknowledge Mr.

Venkateswara Rao, Department of Sericulture, Andhra Pradesh, India for supplying $A$. mylitta larvae. JN acknowledges financial support from Department of Biotechnology, India. AG and AKP are recipients of the fellowship from University Grants Commission (UGC) and Council of Scientific and Industrial Research (CSIR), India respectively.

\section{References}

I. Kanost MR, Jiang H, Yu XQ: Innate immune responses of a lepidopteran insect, Manduca sexta. Immunol Rev 2004, 198:97-105.

2. Cerenius L, Soderhall K: The prophenoloxidase-activating system in invertebrates. Immunol Rev 2004, 198: | 16-126.

3. Steiner H: Peptidoglycan recognition proteins: on and off switches for innate immunity. Immunol Rev 2004, 1 98:83-96.

4. Vodovar N, Vinals M, Liehl P, Basset A, Degrouard J, Spellman P, Boccard $F$, Lemaitre $B$ : Drosophila host defense after oral infection by an entomopathogenic Pseudomonas species. Proc Natl Acad Sci U S A 2005, 102(32): | |4|4-| | 4 | 9.

5. Johansson KC, Metzendorf C, Soderhall K: Microarray analysis of immune challenged Drosophila hemocytes. Exp Cell Res 2005, 305(I): I45-155.

6. Xu X, Dong Y, Abraham EG, Kocan A, Srinivasan P, Ghosh AK, Sinden RE, Ribeiro JM, Jacobs-Lorena M, Kafatos FC, Dimopoulos G: Transcriptome analysis of Anopheles stephensi-Plasmodium berghei interactions. Mol Biochem Parasitol 2005, I 42(I):76-87.

7. Aguilar R, Jedlicka AE, Mintz M, Mahairaki V, Scott AL, Dimopoulos $\mathrm{G}$ : Global gene expression analysis of Anopheles gambiae responses to microbial challenge. Insect Biochem Mol Biol 2005, 35(7):709-7I9.

8. Okano K, Shimada T, Mita K, Maeda S: Comparative expressedsequence-tag analysis of differential gene expression profiles in BmNPV-infected BmN cells. Virology 200I, 282(2):348-356.

9. Hultmark D, Engstrom A, Andersson K, Steiner H, Bennich $H$, Boman HG: Insect immunity. Attacins, a family of antibacterial proteins from Hyalophora cecropia. Embo J 1983, 2(4):57|-576.

10. Zhu Y, Johnson TJ, Myers AA, Kanost MR: Identification by subtractive suppression hybridization of bacteria-induced genes expressed in Manduca sexta fat body. Insect Biochem Mol Biol 2003, 33(5):54I-559.

II. Wang J, Xia Q, He X, Dai M, Ruan J, Chen J, Yu G, Yuan H, Hu Y, Li R, Feng T, Ye C, Lu C, Wang J, Li S, Wong GK, Yang H, Wang J, Xiang Z, Zhou Z, Yu J: SilkDB: a knowledgebase for silkworm biology and genomics. Nucleic Acids Res 2005, 33(Database issue):D399-402.

12. Mita K, Kasahara M, Sasaki S, Nagayasu Y, Yamada T, Kanamori H, Namiki N, Kitagawa M, Yamashita H, Yasukochi Y, Kadono-Okuda K, Yamamoto K, Ajimura M, Ravikumar G, Shimomura M, Nagamura Y, Shin IT, Abe H, Shimada T, Morishita S, Sasaki T: The genome sequence of silkworm, Bombyx mori. DNA Res 2004, II(I):27-35.

13. Mita K, Morimyo M, Okano K, Koike Y, Nohata J, Kawasaki H, Kadono-Okuda K, Yamamoto K, Suzuki MG, Shimada T, Goldsmith MR, Maeda S: The construction of an EST database for Bombyx mori and its application. Proc Natl Acad Sci U S A 2003 , I00(24): $|4| 2|-| 4 \mid 26$.

14. Jain D, Nair DT, Swaminathan G], Abraham EG, Nagaraju J, Salunke DM: Structure of the induced antibacterial protein from tasar silkworm, Antheraea mylitta. Implications to molecular evolution. J Biol Chem 200I, 276(44):4|377-4I 382.

15. Shrivastava B, Ghosh AK: Protein purification, cDNA cloning and characterization of a protease inhibitor from the Indian tasar silkworm, Antheraea mylitta. Insect Biochem Mol Biol 2003, 33(10): 1025-1033.

16. $7 / 9$ clusters of A. mylitta. [http://2/0.2/2.2/2.7:9999/PHP/SILKSAT/uniqueseqs/]

17. Roxstrom-Lindquist K, Terenius O, Faye I: Parasite-specific immune response in adult Drosophila melanogaster: a genomic study. EMBO Rep 2004, 5(2):207-212. 
18. Irving P, Troxler L, Heuer TS, Belvin M, Kopczynski C, Reichhart JM, Hoffmann JA, Hetru C: A genome-wide analysis of immune responses in Drosophila. Proc Natl Acad Sci U S A 200I, 98(26): $|5||9-| 5 \mid 24$.

19. Gudmundsson GH, Lidholm DA, Asling B, Gan R, Boman HG: The cecropin locus. Cloning and expression of a gene cluster encoding three antibacterial peptides in Hyalophora cecropia. J Biol Chem 1991, 266(18): II510-11517.

20. Ochiai M, Ashida M: A pattern recognition protein for peptidoglycan. Cloning the cDNA and the gene of the silkworm, Bombyx mori. J Biol Chem 1999, 274( I 7): I I 854-I I 858.

21. Hultmark D, Steiner H, Rasmuson T, Boman HG: Insect immunity. Purification and properties of three inducible bactericidal proteins from hemolymph of immunized pupae of Hyalophora cecropia. Eur J Biochem 1980, I06(I):7-16.

22. Cheng T, Zhao P, Liu C, Xu P, Gao Z, Xia Q, Xiang Z: Structures, regulatory regions, and inductive expression patterns of antimicrobial peptide genes in the silkworm Bombyx mori. Genomics 2006, 87(3):356-365.

23. Nirmala X, Mita K, Vanisree V, Zurovec M, Sehnal F: Identification of four small molecular mass proteins in the silk of Bombyx mori. Insect Mol Biol 200I, 10(5):437-445

24. Schuhmann B, Seitz V, Vilcinskas A, Podsiadlowski L. Cloning and expression of gallerimycin, an antifungal peptide expressed in immune response of greater wax moth larvae, Galleria mellonella. Arch Insect Biochem Physiol 2003, 53(3): I25-I33.

25. Hoffmann JA: The immune response of Drosophila. Nature 2003, 426(6962):33-38

26. Sun SC, Lindstrom I, Boman HG, Faye I, Schmidt O: Hemolin: an insect-immune protein belonging to the immunoglobulin superfamily. Science 1990, 250(4988): 1729-1732.

27. Dziarski R: Peptidoglycan recognition proteins (PGRPs). Mol Immunol 2004, 40(12):877-886.

28. Watson FL, Puttmann-Holgado R, Thomas F, Lamar DL, Hughes M, Kondo M, Rebel VI, Schmucker D: Extensive diversity of Igsuperfamily proteins in the immune system of insects. Science 2005, 309(5742): 1874-1878.

29. Dong Y, Taylor HE, Dimopoulos G: AgDscam, a Hypervariable Immunoglobulin Domain-Containing Receptor of theAnopheles gambiae Innate Immune System. PLoS Biol 2006, 4(7):e229.

30. Veiga $A B$, Ribeiro JM, Guimaraes JA, Francischetti IM: A catalog for the transcripts from the venomous structures of the caterpillar Lonomia obliqua: identification of the proteins potentially involved in the coagulation disorder and hemorrhagic syndrome. Gene 2005, 355: I I-27.

31. Shin SW, Park SS, Park DS, Kim MG, Kim SC, Brey PT, Park HY: Isolation and characterization of immune-related genes from the fall webworm, Hyphantria cunea, using PCR-based differential display and subtractive cloning. Insect Biochem Mol Biol 1998, 28( I I):827-837.

32. Bao Y, Mega K, Yamano Y, Morishima I: cDNA cloning and expression of bacteria-induced HddI I gene from eri-silkworm, Samia cynthia ricini. Comp Biochem Physiol C Toxicol Pharmacol 2003, I 36(4):337-342.

33. Altschul SF, Madden TL, Schaffer AA, Zhang J, Zhang Z, Miller W, Lipman DJ: Gapped BLAST and PSI-BLAST: a new generation of protein database search programs. Nucleic Acids Res 1997, 25(I 7):3389-3402.

34. He YW, Li H, Zhang J, Hsu CL, Lin E, Zhang N, Guo J, Forbush KA, Bevan MJ: The extracellular matrix protein mindin is a pattern-recognition molecule for microbial pathogens. Nat Immunol 2004, 5(I):88-97.

35. Zhang XF, Wang JF, Matczak E, Proper JA, Groopman JE: Janus kinase $\mathbf{2}$ is involved in stromal cell-derived factor-Ialphainduced tyrosine phosphorylation of focal adhesion proteins and migration of hematopoietic progenitor cells. Blood 200I, 97(II):3342-3348.

36. De Gregorio E, Spellman PT, Rubin GM, Lemaitre B: Genome-wide analysis of the Drosophila immune response by using oligonucleotide microarrays. Proc Natl Acad Sci U S A 200I, 98(22): $12590-12595$

37. Hedengren-Olcott M, Olcott MC, Mooney DT, Ekengren S, Geller BL, Taylor BJ: Differential activation of the NF-kappaB-like factors Relish and Dif in Drosophila melanogaster by fungi and Gram-positive bacteria. J Biol Chem 2004, 279(20):2II2I-2 II 27.
38. Abraham EG, Nagaraju J, Salunke D, Gupta HM, Datta RK: Purification and partial characterization of an induced antibacterial protein in the silkworm, Bombyx mori. J Invertebr Pathol I995, 65(I): 17-24.

39. Ewing B, Green P: Base-calling of automated sequencer traces using phred. II. Error probabilities. Genome Res 1998, 8(3): $186-194$

40. The TGICL program . [www.tigr.org]

4I. Altschul SF, Gish W, Miller W, Myers EW, Lipman DJ: Basic local alignment search tool. J Mol Biol 1990, 215(3):403-4I0.

42. Bru C, Courcelle E, Carrere S, Beausse Y, Dalmar S, Kahn D: The ProDom database of protein domain families: more emphasis on 3D. Nucleic Acids Res 2005, 33(Database issue):D2 I2-5

43. SignalP software. [http://www.cbs.dtu.dk/services/SignalP/].

44. TMHMM program. http://www.cbs.dtu.dk/services/TMHMM-2.0/

45. Rozen S, Skaletsky H: Primer3 on the WWW for general users and for biologist programmers. Methods Mol Biol 2000, 132:365-386.

Publish with Bio Med Central and every scientist can read your work free of charge

"BioMed Central will be the most significant development for disseminating the results of biomedical research in our lifetime. "

Sir Paul Nurse, Cancer Research UK

Your research papers will be:

- available free of charge to the entire biomedical community

- peer reviewed and published immediately upon acceptance

- cited in PubMed and archived on PubMed Central

- yours - you keep the copyright
BiolMedcentral 